\title{
Infants' identification of three-dimensional form from transformations of linear perspective
}

\author{
LISA SHAW, BEVERLY RODER, and EMILY W. BUSHNELL \\ Tufts University, Medford, Massachusetts
}

\begin{abstract}
The ability of infants to perceive three-dimensional structure from transformations of linear perspective was investigated in two studies. Infants were habituated to the pattern of linear perspective transformations corresponding to a particular three-dimensional object, and their relative preference for that object as compared with a different three-dimensional object was assessed both before and after habituation. The habituation displays showed the distorting shadow cast by a rotating object and therefore provided only transformations of linear perspective as information specifying three-dimensional form. The pre- and posttest displays involved the actual threedimensional objects and provided binocular, shading, and texture information specifying threedimensional form, but did not provide informative transformations of linear perspective. In Study 1, 6-month-olds showed changes in preference from pre- to posttest that were related to the identity of the object whose shadow they had seen during habituation; 4-month-olds, however, did not show preference changes related to the habituation object. In Study 2, rhythm information that may have served as a basis for responding in Study 1 was eliminated from the test displays. Sixmonth-olds again showed changes in preference that were dependent on their habituation experience. It is concluded that, by 6 months of age, infants are able to perceive object structure from the isolated cue of transformations of linear perspective. The findings are discussed with reference to infants' three-dimensional form perception based on other cues and also with reference to the emergence of certain spatially related moter activities.
\end{abstract}

The ability to perceive the three-dimensional structure of objects and their spatial layout in the environment is essential for effective motor behavior; consequently, depth perception has been a matter of considerable interest for psychologists. The central problem with respect to this matter is that the perception of three-dimensional objects in a three-dimensional space must be derived from retinal images that are represented two-dimensionally. Psychologists have identified a variety of "cues" to depth that apparently mediate this conversion process and, to determine the origins and development of depth perception, the responses of young infants to a number of these cues have been investigated. The evidence, recently reviewed by Banks and Salapatek (1983), indicates that infants are, indeed, sensitive to many of the identified cues to depth by as early as 2-3 months of age. Furthermore, observations of infants' reaching behavior indicate that actual distance relationships (nearness/farness) specified by proximal cues are appreciated by 5 months of age in the case of disparity cues (Gordon \& Yonas, 1976) and

The research discussed was supported in part by funds awarded to Emily W. Bushnell from Tufts University's Biomedical Research Support Grant (NIH 2 S07 RR07179). Portions of the research were submitted by L. Shaw to Tufts University in partial fulfillment of the requirements for the doctoral degree. The authors wish to thank Katina Athans, Lincoln Craton, Lisa Goodman, Leslie Mandel, and Kevin Roberts for their help in collecting data. They also thank Joshua Bacon and Albert Yonas for their useful comments on an earlier draft. Reprint requests should be addressed to Emily W. Bushnell, Department of Psychology, Tufts University, Medford, MA 02155. by 7 months of age in the case of "pictorial" cues, such as linear perspective, occlusion, familiar size, and so forth (Yonas \& Granrud, 1984). Whether young infants can use the proximal cues to which they are sensitive to perceive the three-dimensional form of objects (in addition to their relative distance) is less clearly established.

There is some suggestion in the literature that threedimensional form is first appreciated in the context of motion (compare E. J. Gibson, Owsley, Walker, and Megaw-Nyce, 1979, with Ruff, 1978; see also Ruff, 1982). The results of two recent studies in which binocular information for three-dimensional structure was eliminated particularly indicate the importance of dynamic information for object recognition by infants. Owsley (1983) had her 4-month-old subjects wear a patch over one eye while they were habituated to an object display. On subsequent (binocular) recognition tests, the infants responded to a cube as a novel stimulus relative to a projectively similar wedge if they had seen the wedge oscillating in rotation during habituation, but not if they had seen successive static views of the wedge during habituation. Similarly, Kellman (1984) observed that 4-month-olds responded to one object as novel relative to another when they had seen videotapes of the "familiar" object undergoing rotation prior to the recognition tests, but not when they had seen successive static views taken from the rotation sequences.

There are several possible reasons why infants may extract three-dimensional form more readily from moving displays than from static ones. First, it is possible that 
motion presentations simply enhance attention to identifying information that is also available in sequences of static views. Alternatively, there are two kinds of information that are available only in dynamic displays, and young infants may especially rely on one or both of these for perceiving three-dimensional form (see Ruff, 1980). When an object moves (or when the observer moves with respect to the object), there are transformations in the arrays of visible texture on the surfaces of the object, including the expansion and contraction of surface texture gradients and the accretion and deletion of texture with respect to the background. Such patterns of "optical flow" have been identified by J. J. Gibson $(1966,1979)$ as powerful indicators of spatial relations, and it has been shown that young infants are responsive to them for perceiving distance relations (Ball \& Vurpillot, 1976; Carroll \& E. J. Gibson, 1981; Granrud et al., 1984) and for perceiving two-dimensional form (Kaufmann-Hayoz, Kaufmann, \& Stucki, 1986). With object motion, there are also transformations of the two-dimensional contour projected onto the retina. Such transformations of linear perspective may be thought of as a special, reduced case of optical flow, in which only elements of texture at the projected edges of the stimulus are salient. The pattern of contour transformations when a particular threedimensional form rotates (or when one moves around the form) is generally unique to that form, and it has been shown that human adults are extremely sensitive to this sort of information for three-dimensional form (J. J. Gibson, 1966; Johansson, 1974; Wallach \& O'Connell, 1953). Exhibiting what Wallach and O'Connell termed the "kinetic depth effect" (KDE), subjects are able to quickly and accurately identify rotating three-dimensional forms from viewing the transformations of their shadows, provided the objects are positioned so that the contour lines of the shadows change both in length and relative angle.

Kellman and Short (1985) attempted to determine whether young infants could perceive three-dimensional form from transformations of linear perspective isolated from all other cues, as in the KDE. They used videotape displays of rotating wire outlines of forms to eliminate the surface texture transformations that may have been informative in the Owsley (1983) and Kellman (1984) studies. Four-month-olds distinguished a novel wire outline from a familiar one after they had been habituated to a display of the familiar form undergoing rotation, but not after habituation to a series of static views of the familiar form. Kellman and Short concluded from these results that 4-month-olds could extract three-dimensional form from transformations of linear perspective alone. However, it is possible that the infants in Kellman and Short's study were responding on the basis of proximal, two-dimensional information rather than on the basis of three-dimensional form. The two wire outlines used in the study each consisted of two triangles sharing a side. In one case, the outline was "bent" along the common side at an angle of $165^{\circ}$; in the other case, it was bent at an angle of $95^{\circ}$. Thus, as the one form underwent rotation, its projection would at one point show two lines meeting at a very obtuse angle, whereas at the analogous point in rotation, the projection of the other form would show two lines meeting at almost a right angle. Since young infants can discriminate angles of different degrees (Cohen \& Younger, 1984), Kellman and Short's infants could have been dishabituating to the change in twodimensional angle that accompanied the novel threedimensional form rather than to the change in form itself. The proximal cue of angle was also available in the static displays with which infants evidenced no dishabituation, but infants may simply have been less attentive and therefore less sensitive to the available information in the no-motion condition.

In the research reported below, we sought to determine whether infants could perceive three-dimensional structure from patterns of linear perspective transformation alone. To circumvent the proximal-cue interpretation possible for the results of prior research, we used the "transfer" method advocated by Yonas and Pick (1975). Infants were habituated to a KDE display that contained only transformations of linear perspective as information for three-dimensional structure. All other cues for three-dimensional structure were eliminated from the habituation displays by using the shadow-casting technique of Wallach and O'Connell's classic KDE work. The three-dimensional structures of the test stimuli, on the other hand, were specified by many sources of information, including binocular disparity, texture transformations, shading changes, and so forth, but not by linear perspective transformations; informative contour transformations were specifically eliminated from the test displays. Thus, any influence of the habituation experience on test performance could not be based on a matching of proximal, two-dimensional pattern information, but would have to be based on the perception of a higher order property to which the different proximal variables were each related, such as the distal stimulus of threedimensional structure.

\section{STUDY 1}

\section{Method}

Subjects. Twenty 4-month-old infants (mean age $=119.6$ days, $S D=3.5$ ) and 206 -month-old infants (mean age $=176.3$ days, $S D=2.7$ ) served as subjects in Study 1 . There were 10 males and 10 females in each age group. An additional 16 infants were tested but were not included in the final sample. Three infants were excluded because of experimenter error or equipment failure, 1 because of fussing, and 12 because of insufficient attention to the stimuli during habituation. All of the subjects were recruited from a predominantly white middle-class population, and all were fullterm and in apparent good health.

Apparatus. Two display booths were used in Study $1-$ one for the presentation of habituation trials and one for the presentation of test trials. The habituation booth consisted of a table on which a three-sided enclosure was situated. The two side panels of the enclosure were made of gray Triwall cardboard; they served to screen from the infants' view the experimental equipment and other 
potentially distracting features of the laboratory room. The center panel was a $90 \times 61 \mathrm{~cm}$ Polacoat screen, behind which a slide projector was positioned. Just behind the screen, between it and the projector, was a $15 \times 15 \mathrm{~cm}$ wooden platform that was mounted on ball bearings and tilted toward the screen at an angle of about $6^{\circ}$. Attached to this platform was a wooden handle with which the platform could be rotated. A stimulus object could be fixed onto a peg at the center of the platform so that when the slide projector was turned on, the object's shadow would be cast upon the screen.
From the front, the display appeared as a black two-dimensional form centered in the "window" of light thrown on the screen by the slide projector (see Figure lc).

The test booth also consisted of a table on which a three-sided "surround" was situated. This booth was positioned adjacent to the habituation booth and at right angles to it. Rather than a projection screen, the center panel of the test booth was a white matboard panel into which a $15 \mathrm{~cm}$ high $\times 28 \mathrm{~cm}$ wide window had been cut. Just behind this window was a second rotatable platform onto

\section{Rectangle}

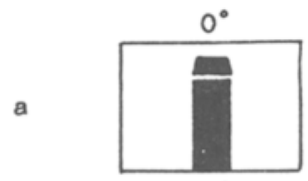

$\mathrm{O}^{\bullet}$

b

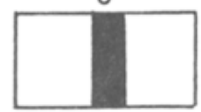

$0^{\bullet}$

c

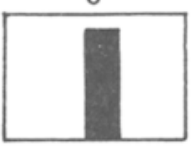

$+6.5 \mathrm{~cm}$
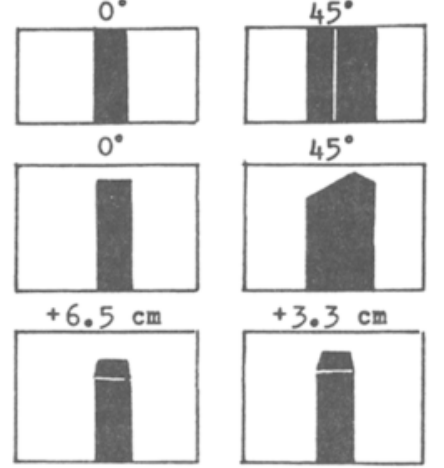

$5^{\circ}$

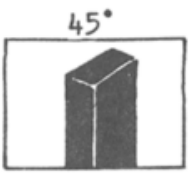

$45^{\circ}$
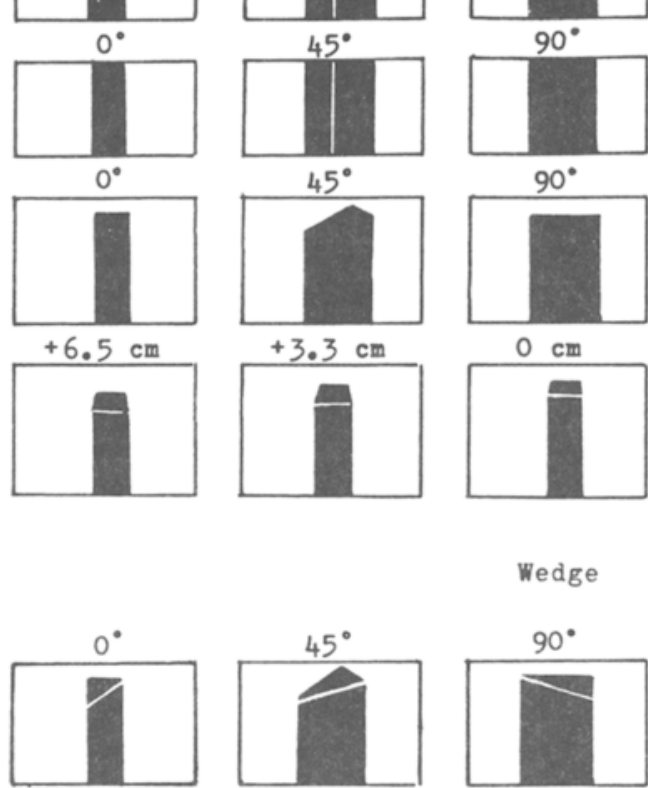

$0^{\circ}$

b

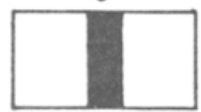

$0^{\circ}$

c

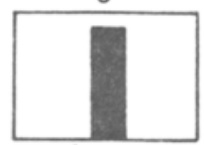

$+6.5 \mathrm{~cm}$

d

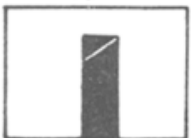

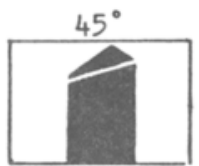

$45^{\circ}$

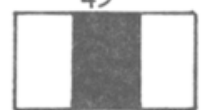

$45^{\circ}$

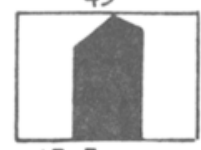

$+3.3 \mathrm{~cm}$

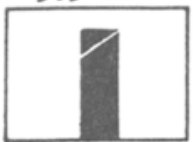

$90^{\circ}$

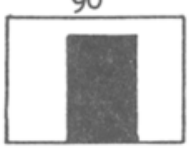

$0 \mathrm{~cm}$

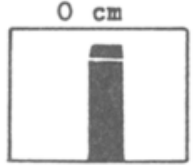

Wedge

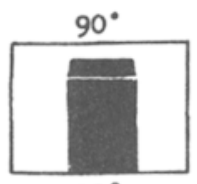

$90^{\circ}$

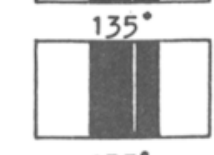

$135^{\circ}$

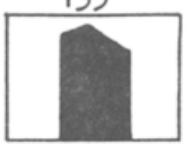

$-3.3 \mathrm{~cm}$
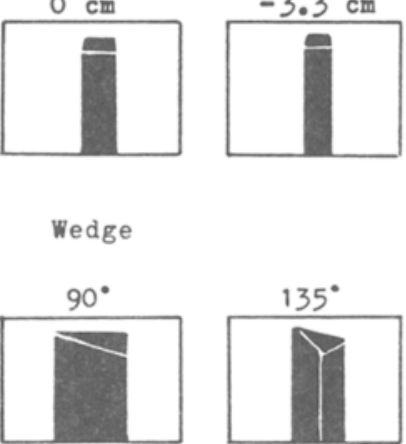

$90^{\circ}$

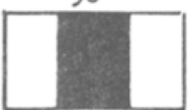

$90^{\circ}$

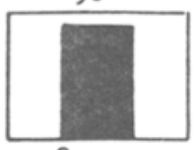

$0 \mathrm{~cm}$

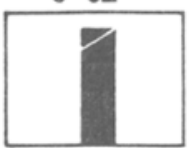

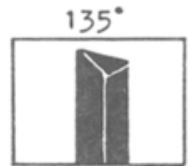

$135^{\circ}$

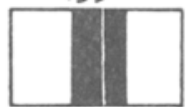

$135^{\circ}$

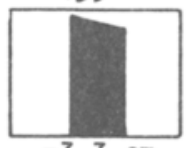

$-3.3 \mathrm{~cm}$

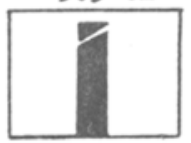

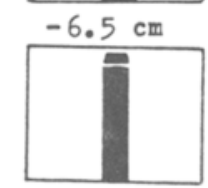

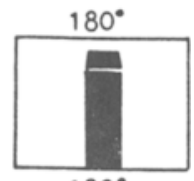

$180^{\circ}$

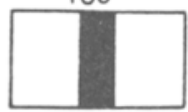

$180^{\circ}$
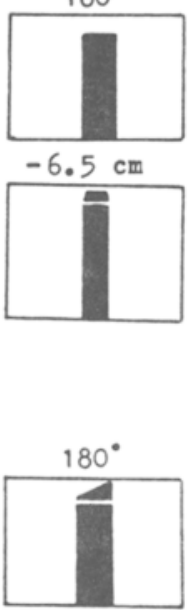

$180^{\circ}$

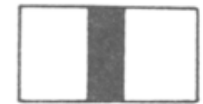

$180^{\circ}$
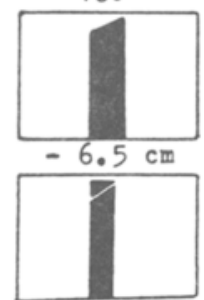

Figure 1. Stimuli used in Studies 1 and 2, depicted at selected points between the starting position and the maximum displacement of a single oscillation. Figure shows: (a) the three-dimensional stimuli as they would appear during rotation if fully displayed and fully illuminated; (b) the three-dimensional stimuli as they appeared during rotation in the pre- and posttest trials in Study 1 ; (c) the two-dimensional shadows of the rotating stimuli as they appeared during the habituation trials in Studies 1 and 2; (d) the threedimensional stimuli as they appeared when withdrawn and advanced during the pre- and posttest trials in Study 2. For the stimulus presentations illustrated in a, b, and $c$, the $0^{\circ}$ position represents the starting point of the rotation for each trial. For the stimulus presentations illustrated in $d$, the $b-\mathrm{cm}$ position represents the starting point of the backward/forward movement. The white lines shown in $a, b$, and $d$ represent edges visible from shading and other cues available in the test displays; no such white lines were actually present on the stimuli. 
which stimulus objects could be fastened. The platform was enclosed from behind by a semicircular "baffle"' made of white matboard; the baffle blocked from view everything behind the stimulus object. A small spotlight above the platform illuminated the object below. From the front, the display appeared as a dark form centered in the window of white created by the cutout in the center panel and the baffle surrounding the object (see Figure 1b). The display was comparable in coloring and size to the habituation display, except that the form in the center was actually a threedimensional object rather than the two-dimensional shadow of one. Another important difference was that the window in the test booth was not as high as the window of light in the habituation display, so the top portion of the object was screened from view.

At the bottom of the center panels of both booths were two small apertures, through which observers stationed behind the booths could watch the infants. The observers recorded the infants' looking behavior by operating pushbuttons interfaced with a Commodore PET microprocessor.

Stimulus displays. The stimuli used in Study 1 were two wooden blocks, each painted flat black. One block was a rectangular solid, $20 \mathrm{~cm}$ tall, $5 \mathrm{~cm}$ across the narrower two sides, and $10 \mathrm{~cm}$ across the wider two sides. The second block was a wedge-shaped solid made by bisecting the rectangular solid lengthwise along the diagonal formed by the $5 \times 10 \mathrm{~cm}$ sides. Thus, the wedge was also $20 \mathrm{~cm}$ tall, and it measured $5 \mathrm{~cm}$ across the narrowest of its three sides and $10 \mathrm{~cm}$ across the side at right angles to the narrowest side. The wedge was made from the rectangle as described so that the minimum and maximum heights and widths of the shadows cast by the two stimuli would be equivalent.

At the start of both test and habituation trials, the stimulus object, whether the rectangle or the wedge, was fixed on the platform so that a (the) $5-\mathrm{cm}$-wide edge was at the back of the platform and parallel to the frontoparallel plane of the infant. The twodimensional projections of the two objects when so positioned are identical, as is shown in the first $\left(0^{\circ}\right)$ panels of Figure $1 \mathrm{c}$. The platform was then rotated clockwise through $180^{\circ}$; the two-dimensional projections of the objects in the latter position are shown in the final $\left(180^{\circ}\right)$ panels of Figure 1c. Projections for positions intermediate to the starting position and the $180^{\circ}$ position are shown in the several middle panels of Figure 1c.

During the test and habituation trials, the platform was continuously rotated back and forth between the starting position and the $180^{\circ}$ position at a rate of about 1 complete oscillation every $4 \mathrm{sec}$. Since the stimulus object was tilted, the contour lines at the top of the habituation display changed in both length and angle with respect to one another as the object casting the visible shadow was rotated. Adults viewing the habituation displays could readily identify the stimulus object as either the rectangle or the wedge from the pattern of linear perspective transformations provided, instantiating the KDE. Because the test booth did not allow the top of the stimulus object to be seen, similarly informative patterns of linear perspective transformations were not available when the test displays were viewed; the projections of both stimuli simply expanded and contracted in width (see Figure 1b). However, changes in surface texture gradients and shading cues, though minimal given the smooth black stimuli and the overhead lighting arrangement, were sufficient for adults viewing the test displays monocularly to discriminate the rectangle and wedge. With binocular viewing, as in the procedure with infants, the test displays were very quickly and very easily discriminable for adults. The texture, shading, and disparity cues that enabled identification of the test displays were of course not present in the habituation displays, since they involved shadow-casts.

Procedure. The experimental procedure consisted of three phases: a pretest phase, a habituation phase, and a posttest phase. A withinsubject, comparison-to-baseline design was employed in order to neutralize the influence of any strong spontaneous preferences for one or the other of the two stimuli. For the pretest phase, the infant was seated on a parent's lap facing the matboard center panel of the test booth, about $55 \mathrm{~cm}$ away from it. The height of the infant above the floor was adjusted so that neither the tops nor the bottoms of the stimulus objects would be visible from the infant's viewing angle. When the infant was settled at the proper height, the room lights were dimmed and the experimenters positioned themselves behind the enclosure.

For each infant, one or the other of the stimulus objects was designated as the "familiar" stimulus, that is, the one that would be involved during the habituation phase. For 10 of the infants in each age group, the rectangle was the "familiar" stimulus; for the other 10 , it was the wedge. There were three pretest trials. For 5 of the babies in each age $\times$ habituation-object group of 10 , the "familiar" object was presented on the first and third trials and the other object was presented on the second trial; for the other 5 babies in each group, the "familiar" object was presented on the second trial and the other object was presented on the first and third trials.

To initiate each pretest trial, the appropriate object was positioned on the platform and the spotlight above it was turned on, revealing the stimulus through the window of the booth. One of the experimenters behind the booth oscillated the platform, while a second experimenter looked through the aperture and monitored the infant's visual fixations. The observer was uninformed as to which one of the stimulus objects was involved on each trial. The onset and duration of each pretest trial was controlled by the infant's looking behavior; each trial began when the infant first fixated the stimulus and ended when no fixation toward the stimulus had been recorded for 2 consecutive seconds. When the trial ended, the spotlight was turned off for about $10 \mathrm{sec}$, while the stimulus object was changed for the next trial.

Following the three pretest trials, the chair on which the parent and infant sat was swiveled to face the habituation display booth; the infant was again about $55 \mathrm{~cm}$ away from the center panel, in this case the Polacoat screen. The experimenters moved to behind the habituation booth and positioned the "familiar" stimulus object on the platform behind the projection screen. A series of habituation trials was then presented. To initiate each habituation trial, the slide projector was turned on, revealing the shadow of the stimulus to the infant. The experimenter who had rotated the platform during the pretests again rotated the platform with the object casting the shadow, while the second experimenter again monitored the infant's visual fixations. The onset and termination of each habituation trial was infant-controlled according to the criteria used in the pretest trials. When each habituation trial terminated, the slide projector was turned off for about 3 to $5 \mathrm{sec}$ while the stimulus object was rotated back to the $0^{\circ}$ position. Habituation trials were presented until the duration of looking for any one trial was less than or equal to the duration of looking recorded for either the first or second habituation trial, whichever was longer. Thus, at least three habituation trials were presented to each infant. Infants who did not look at the stimulus display for at least $15 \mathrm{sec}$ during either the first or second habituation trial were excluded from the study $(n=12)$.

When the habituation criterion had been met, the infant was turned to face the test booth again for the posttest phase. During this part of the procedure, the two stimulus objects were presented in exactly the same alternating order and in the same manner as during the pretest phase, on the assumption that any effects of order or spontaneous preferences for either the rectangle or the wedge would therefore influence the infant's behavior similarly during the preand posttest phases of the procedure. Thus, any systematic difference between the infants' relative attention to the two objects during the posttest phase and that during the pretest phase could be attributed to the intervening habituation experience.

To enhance the subjects' attention to the visual displays, all of the trials (pretest, habituation, and posttest) were accompanied by 
a tape recording of a female voice reading from a novel (see Owsley, 1980). During the procedure for 3 additional infants, a second observer also monitored visual behavior. To assess interobserver reliability, the Pearson product-moment correlation procedure was run on the two durations of looking recorded for each trial monitored by two observers; the resulting correlation was .99 .

\section{Results and Discussion}

It was observed during the data collection that the infants were generally rather restless and inattentive during the third pre- and posttest trials, and preliminary analyses indicated that the infants in the various test-order and habituation-object conditions behaved equivalently on these trials. Further analyses of test-trial behavior were therefore conducted using only the looking times for the first and second pre- and posttest trials. Analyses of variance conducted to determine whether infants in the two age groups and in the various test-order and habituationobject conditions behaved similarly prior to the test trials yielded no significant main effects or interactions with respect to the looking times for the two pretest trials (means for the pretest trials are displayed in Table 1) or with respect to the numbers of habituation trials to criterion (grand mean $=3.3$ ). However, an ANOVA on the total looking times accumulated during habituation yielded a main effect of age $[F(1,32)=7.35, p<.025]$. The habituation display was looked at longer by the 4month-old infants $(M=98.8 \mathrm{sec}, S D=56.4)$ than by the 6-month-old infants $(M=56.6 \mathrm{sec}, S D=36.7)$. Since the habituation experiences of the 4-month-olds and the 6-month-olds were not equivalent, separate planned comparisons for examining the effect of the habituation experience on test-trial behavior were conducted for the two age groups.

The mean looking times to the "familiar" stimulus and to the other stimulus on the pretest trials and on the posttest trials are shown for each age group in Table 1. To examine the effect of the habituation experience, the infants' behavior on the posttest trials was compared with their behavior on the pretest trials. For each infant, "preference ratios" were computed for the pretest phase and for the posttest phase by dividing the looking time allocated to the other stimulus during each phase by the sum of the looking times to the "familiar" stimulus and to the other stimulus during that phase. The resulting ratios for each age group and each phase are shown in Table 1. For each age group, the pre- and posttest ratios were then compared with a two-tailed correlated $t$ test. As Table 1 shows, for the 6-month-olds, the preference for the other stimulus as compared with the "familiar" stimulus was significantly greater during the posttest phase than during the pretest phase; for the 4-month-olds, the preference ratios for the two phases were not significantly different. These findings are corroborated by an analysis of the directions of preference shift for individual infants. Fifteen of the 20 6-month-olds (two-tailed binomial $p=.042$ ) but only 9 of the 20 4-month-olds (two-tailed binomial $p>.50$ ) showed a greater preference for the other stimulus during the posttest phase than during the pretest phase. Furthermore, 2 (test order) $\times 2$ (habituation object) $\times 2$ (phase-pre- or posttest) $\times 2$ (trial"familiar" object or other object) ANOVAs conducted on the looking times for the pre- and posttest trials for each age group yielded a significant phase $\times$ trial interaction for the 6-month-olds $[F(1,16)=4.56, p<.05]$ but not for the 4-month-olds. The responses of the 6-month-olds to the "familiar" and other objects were not significantly different on the pretest, but were different at a borderline level of significance on the posttest $[F(1,19)=4.02, p<$ $.06]$. Thus, the results of the planned comparisons on the preference ratios, the analyses of individual preference shifts, and ANOVAs on the pre- and posttest looking times all indicate that the posttest behavior was affected by the identity of the habituation stimulus for the 6-month-olds but not for the 4-month-olds. ${ }^{1}$ As the data in Table 1 show, the pre- vs. posttest comparisons for the 4-month-olds were on the average in the same direction as those for the 6month-olds, but they were not as strong and they involved somewhat greater variance for the posttest phase.

Consideration of the pretest behavior of the 6-montholds raised the concern that their posttest behavior may have been influenced by their particular pretest experience rather than by the habituation phase experience. Since the infants on the average tended to look longer at the "familiar" stimulus during the pretest (see Table 1), it might be argued that they became relatively more familiar

Table 1

Mean Looking Times and Preference Ratios for the Pretest and Posttest Phases

\begin{tabular}{|c|c|c|c|c|c|c|c|c|c|}
\hline \multirow[b]{3}{*}{ Study } & \multirow{3}{*}{$\begin{array}{c}\text { Subject } \\
\text { Age }\end{array}$} & \multirow[b]{3}{*}{ Test } & \multicolumn{4}{|c|}{ Looking Time (in Seconds) } & \multirow{2}{*}{\multicolumn{2}{|c|}{ Preference Ratio }} & \multirow[b]{3}{*}{ Correlated $t$} \\
\hline & & & \multicolumn{2}{|c|}{ "Familiar" Object } & \multicolumn{2}{|c|}{ Other Object } & & & \\
\hline & & & Mean & $S D$ & Mean & $S D$ & Mean & $S D$ & \\
\hline \multirow[t]{2}{*}{1} & 4 months & $\begin{array}{l}\text { Pre } \\
\text { Post }\end{array}$ & $\begin{array}{l}29.3 \\
10.4\end{array}$ & $\begin{array}{r}42.2 \\
8.1\end{array}$ & $\begin{array}{l}20.5 \\
17.3\end{array}$ & $\begin{array}{l}22.6 \\
28.9\end{array}$ & $\begin{array}{l}.49 \\
.53\end{array}$ & $\begin{array}{l}.22 \\
.21\end{array}$ & -.65 \\
\hline & 6 months & $\begin{array}{l}\text { Pre } \\
\text { Post }\end{array}$ & $\begin{array}{l}35.8 \\
12.9\end{array}$ & $\begin{array}{r}46.2 \\
9.0\end{array}$ & $\begin{array}{l}19.9 \\
18.9\end{array}$ & $\begin{array}{l}14.1 \\
18.0\end{array}$ & $\begin{array}{l}.44 \\
.57\end{array}$ & $\begin{array}{l}.20 \\
.17\end{array}$ & $-2.34^{*}$ \\
\hline 2 & 6 months & $\begin{array}{l}\text { Pre } \\
\text { Post }\end{array}$ & $\begin{array}{l}11.2 \\
10.9\end{array}$ & $\begin{array}{l}5.0 \\
7.5\end{array}$ & $\begin{array}{r}12.8 \\
7.2\end{array}$ & $\begin{array}{l}7.5 \\
2.9\end{array}$ & $\begin{array}{l}.52 \\
.42\end{array}$ & $\begin{array}{l}.12 \\
.15\end{array}$ & $2.63+$ \\
\hline
\end{tabular}

Note-The "familiar" object was the object (rectangle or wedge) whose shadow was presented during the habituation phase. $N=20$ for each of the three groups. Preference ratio = looking time to the other stimulus/(looking time to the other stimulus + looking time to the familiar stimulus). $\quad *_{p}<.05$, two-tailed. $\quad t p<.02$, two-tailed. 
with this stimulus and therefore preferred the other stimulus during the posttest. To assess this possibility, the data for the 6-month-olds were further examined with an analysis taking into account which stimulus was preferred by each infant on the pretest. The 6-month-old subjects were divided into infants who preferred the "familiar" stimulus on the pretest $(n=12)$ and infants who preferred the other stimulus on the pretest $(n=8)$. For each infant, the relative preference on the posttest for the stimulus that was not preferred on the pretest was noted, and the values of this variable for the two groups were compared. If only the relative amounts of looking at the stimuli on the pretest affected the relative amounts of looking on the posttest, no difference between the two groups would be expected. Infants who preferred the "familiar" stimulus on the pretest would be expected to favor the other stimulus on the posttest to the same extent that infants who preferred the other on the pretest would favor the "familiar" on the posttest. In fact, though, when the two groups were compared with respect to their posttest behavior toward the stimulus not preferred on the pretest, they did not behave equivalently $[t(18)=1.81, .05<$ two-tailed $p<.1$ ]. Infants who preferred the "familiar" stimulus on the pretest did, indeed, prefer the other stimulus on the posttest (posttest $M=.57, S D=.19$ ), but infants who preferred the other stimulus on the pretest did not analogously shift their preference to the "familiar" stimulus (posttest $M=.43, S D=.15$ ). Thus, on the posttest, infants preferred the stimulus whose shadow they had not seen during habituation, whether or not they had looked at that stimulus longer on the pretest. It may therefore be concluded that the 6-month-olds' relatively stronger preference for the other stimulus on the posttest as compared with the pretest was effected by the habituation phase experience, not by the pretest experience. The same sort of analysis was also conducted for the 4-month-olds. In this case, infants who initially preferred the "familiar" stimulus $(n=8)$ and infants who initially preferred the other stimulus $(n=12)$ did not behave differently with respect to their posttest preference for the initially nonpreferred stimulus [posttest $M \mathrm{~s}=.54$ and .47 , respectively, $t(18)=.69, p>.25]$. Thus, once again, there was no evidence that the 4-month-olds' posttest behavior was systematically influenced by the habituation phase experience.

The results of Study 1 indicate that, by 6 months of age, infants can make use of the information related to threedimensional form that is available in KDE displays. After viewing a KDE display that specified one or the other of two three-dimensional forms, 6-month-olds showed a relative preference that was different from a corresponding baseline measure for the object that was not involved in the KDE display. Four-month-olds, though, did not consistently evidence a preference shift related to their viewing of the KDE display, and thus they provided no evidence for sensitivity to the information available in the display.

There are several possible explanations for the indefinite posttest behavior of the 4-month-olds. First, the 4-month- olds may simply have been fatigued and hence relatively inattentive by the time they were presented with the posttest trials. Alternatively, the 4-month-olds may not have been able to make use of the information in the distorting shadows that specified three-dimensional form. That is, linear perspective transformations in isolation may not be a sufficient cue to three-dimensional form for 4-montholds. In previous studies in which 4-month-olds have apparently perceived three-dimensional form from displays containing linear perspective transformations, other sorts of information related to object structure were also available. These included transformations in texture and shading in the Owsley (1983) and Kellman (1984) studies and a two-dimensional characteristic (angle) in the Kellman and Short (1985) study. Our negative results with 4month-olds suggest that the young infants in these prior studies may have relied on such additional cues instead of, or perhaps in conjunction with, transformations of linear perspective. Finally, it is possible that the 4-montholds in Study 1 perceived the three-dimensional form of the object whose shadow they were presented during habituation but could not readily identify the objects during the test trials. During the test trials, the threedimensional forms of the objects were specified most clearly by binocular disparity, although subtle texture flow and shading cues were also available. Recent research indicates that sensitivity to binocular disparity is just emergent at 3 to 4 months of age, so that some 4-month-olds reliably detect disparity whereas others do not (Birch, Gwiazda, \& Held, 1982; Birch, Shimojo, \& Held, 1985; Fox, Aslin, Shea, \& Dumais, 1980). Thus, it is probable that a number of our 4-month-olds were unable to perceive object structure from the information available on the test trials and therefore had no way to demonstrate successful perception of object structure during habituation. The random performance of these infants would, of course, dilute any more systematic behavior on the part of infants who perceived object structure under both conditions, thereby accounting for the negative results for the 4-month-olds overall.

The last interpretation discussed above seems especially likely in view of some recent work by Yonas, Arterberry, and Granrud (1986). They tested 4-month-old infants for the perception of object structure from linear perspective transformations with a transfer procedure similar to ours, and they also independently assessed each infant's sensitivity to binocular disparity. Infants who responded consistently in the disparity detection test provided evidence that they perceived three-dimensional form from linear perspective transformations in the transfer procedure, whereas infants who failed to respond consistently in the disparity detection test did not. It is uncertain whether the disparity-insensitive infants in the Yonas et al. study (and those presumably in our 4-month-old sample) were able to perceive three-dimensional form from transformations of linear perspective and just unable to show it in the transfer paradigm or whether they were immature with respect to depth perception from kinetic information as well as 
with respect to disparity sensitivity. In this regard, it is provocative that, in Study 1, the 4-month-olds accumulated more time looking at the KDE displays before habituating than the 6-month-olds did. Perhaps their longer interest reflects difficulty in assimilating the display (i.e., in perceiving three-dimensional form from it) for the younger infants.

In contrast to the 4-month-olds, who may be at a transitional age, the 6-month-olds in Study 1 provided clearcut results. Their test behavior was systematically related to the identity of the one object that corresponded to the distorting shadow they had seen in a KDE display during the habituation phase. The most immediate interpretation of this result is that 6-month-old infants respond to displays of linear perspective transformations in a manner similar to that of adults. That is, it seems likely that the infants perceived the three-dimensional form of the habituation object from the distorting two-dimensional pattern they were presented. Presumably, they also perceived the three-dimensional forms of the test objects from the binocular cues and other depth information available in the test displays; the recognition of one of these as having the same form they had perceived during habituation then influenced their relative attention on the posttest trials.

There is another possible explanation for the effect of the habituation experience on the 6-month-olds' posttest behavior, however. Since the platform was rotated at an essentially constant rate across all trials, angles emerged and disappeared in the habituation display in a characteristic rhythm for the rectangle and in a different characteristic rhythm for the wedge. Angles could not similarly be seen emerging and disappearing in the test displays, since the top of the object was screened, but the edges of the objects could be seen passing by in related rhythms. Thus, the infants' posttest preference for the other object over the familiar one could have been based on their noting a correspondence between the rhythm in which angles emerged during habituation and the rhythm in which edges went by during the test trial with the familiar object. To determine whether the sensitivity to kinetic information demonstrated in Study 1 was based on the perception of three-dimensional structure as opposed to matching proximal rhythms, a second study was conducted in which the related rhythmic information was eliminated from the test displays.

\section{STUDY 2}

\footnotetext{
Method

Subjects. Ten female and 10 male 6-month-old infants who had not participated in Study 1 served a subjects in Study 2 (mean age $=177.6$ days, $S D=6.2$ ). An additional 9 infants were tested but not included in the final sample. Four infants were excluded because of experimenter error or equipment failure, and 5 were excluded because of fussing. All aspects of subject recruitment were identical to those used in Study 1.
}

Apparatus and Stimulus displays. The apparatus and stimulus displays used in Study 2 were the same as those used in Study 1, except for the specific nature of the pre- and posttest displays. In Study 2 , the objects were not rotated during the pre- and posttest trials, but instead were moved back and forth in depth. These displays retained the attention-attracting feature of motion from the Study 1 displays, but eliminated the information of edges passing by in rhythms corresponding to those perceivable in the habituation displays. The habituation displays for Study 2 were the same shadow-casts of the rotating wedge and rectangle used in Study 1.

At the start of the test trials in Study 2, the stimulus object, whether the rectangle or the wedge, was fixed on a platform in the same position as described for Study 1. The test platform in Study 2 was located behind the screen and tilted forward as in Study 1, but it was not rotatable. Instead, the platform was set within two wooden guides that extended $13 \mathrm{~cm}$ back perpendicularly from the center panel of the test booth. A wooden rod was connected to the back of the platform and extended out behind the baffle; with the rod, the platform could be moved back and forth along the track created by the guides. At the start of the trials, the platform was positioned at the midpoint of the track; during the trials, it was moved back and forth at a steady rate of about 1 complete oscillation every $4 \mathrm{sec}$. The window in the center panel of the test booth for Study 2 measured $20 \mathrm{~cm}$ high $\times 28 \mathrm{~cm}$ wide, allowing the top of the object to be seen. Since the object was not rotated during the test trials, full view of its top did not provide transformations of linear perspective corresponding to those presented during the habituation phase. As in Study 1, the three-dimensional forms of the test objects were specified predominantly by binocular disparity and also by texture cues, shading cues, and other static depth cues not available in the KDE display. Thus, the transfer aspect of the design was again incorporated in Study 2, but with the additional control provided by the elimination of rhythm information as a possible proximal basis for responding. The test displays used in Study 2 are illustrated in Figure 1d.

Procedure. The procedure used in Study 2 was very similar to that used in Study 1, again incorporating a pretest phase, a habituation phase, and a posttest phase. However, in the pre- and posttest phases for Study 2, there were only two trials (rather than three), one involving each of the two stimuli. The stimulus designated as "familiar" (wedge or rectangle) was counterbalanced across the 20 subjects, and the order in which the two stimuli ("familiar" and other) were shown on the pre- and posttest trials was counterbalanced within each "familiar" object group.

In Study 2, each test trial began when the spotlight had been turned on and the infant first looked at the stimulus. After the infant had fixated the stimulus for approximately $5 \mathrm{sec}$, an experimenter behind the booth began to move the stimulus, alternately withdrawing and then advancing it. Each test trial continued for at least $15 \mathrm{sec}$ after its onset, regardless of the looking behavior of the infant. The trial then continued beyond this minimum exposure period until no stimulus fixation had been recorded for 2 consecutive seconds. This combination of fixed length and infant-controlled termination of the test trials was incorporated on the basis of its usefulness in research on another issue (see Bushnell \& Roder, 1985). The procedure for the habituation phase was exactly the same as that used in Study 1, except that infants who did not look for at least $15 \mathrm{sec}$ on either the first or second habituation trial were not excluded. Instead, habituation trials continued until such infants looked for at least $15 \mathrm{sec}$ on some given trial and then habituated to criterion with respect to that trial, or until 10 habituation trials had been presented. During the trials of all three phases, one experimenter manipulated the stimuli while a second experimenter monitored the infant's visual fixations. For 8 of the 20 infants in Study 2, looking behavior was monitored by two observers. To assess interobserver reliability, 
the Pearson product-moment correlation procedure was run on the two durations of looking recorded for each trial monitored by 2 observers; the resulting correlation was .99 .

\section{Results and Discussion}

Analyses of variance conducted to determine whether infants in the various habituation-object and test-order conditions behaved similarly prior to the test trials yielded no significant main effects or interactions with respect to the looking times for the two pretest trials (means for the pretest trials are displayed in Table 1), the numbers of habituation trials to criterion (grand mean $=4.65, S D$ $=2.41$ ), or the total looking times accumulated during habituation (grand mean $=73.65 \mathrm{sec}, S D=41.1$ ). The mean looking times for the infants in Study 2 to the "familiar" stimulus and to the other stimulus on the preand posttest trials are shown in Table 1 . To examine the effect of the habituation experience, the infants' posttest behavior was compared with their pretest behavior using a two-tailed correlated $t$ test on preference ratios computed as in Study 1. The comparison between the mean preference ratios for the two phases is indicated in Table 1 . As the table shows, the preference for the other stimulus over the "familiar" stimulus during the posttest phase was significantly different from the preference shown during the pretest phase. Note that the preference shift demonstrated from pre- to posttest in Study 2 is at least as strong as that demonstrated by the 6-month-olds in Study 1, but it is in the opposite direction; this reversal will be considered in the General Discussion below. As in Study 1, the data for Study 2 were also analyzed according to the direction of preference shift for each individual infant. Fourteen of the 19 infants who showed a change of preference (one infant's pre- and posttest preference ratios were tied) showed a greater preference for the "familiar" stimulus on the posttest than on the pretest (two-tailed binomial $p=.064$ ). Finally, a 2 (habituation object) $\times 2$ (test order) $\times 2$ (phase) $\times 2$ (trial) ANOVA conducted on the looking times for the pre- and posttest trials yielded a significant phase $\times$ trial interaction $[F(1,16)=6.60, p<.025]$. The infants' responses to the "familiar" and other objects were not significantly different on the pretest, but were significantly different on the posttest $[F(1,19)=6.85, p<.025]$. Thus, the results of the planned comparison on the preference ratios, the analysis of individual preference shifts, and the ANOVA on the test-trial looking times all indicate that the infants' posttest behavior was affected by the identity of the habituation stimulus.

As in Study 1, the infants in Study 2 were divided into two groups, according to whether they preferred the "familiar" stimulus $(n=8)$ or the other stimulus $(n=12)$ on the pretest. These two groups were then compared with respect to their preference on the posttest for the stimulus that was not preferred on the pretest. The behavior of the two groups was not equivalent $[t(18)=2.48$, two- tailed $p<.05]$. Both infants who preferred the "familiar" stimulus on the pretest and infants who preferred the other stimulus on the pretest preferred the "familiar" stimulus on the posttest (posttest means for the initially nonpreferred stimulus $=.38$ and .55 , respectively, $S D \mathrm{~s}=.16$ and .14). Thus, as in Study 1, the infants' altered preference for the other stimulus on the posttest relative to that on the pretest was evidently effected by the habituation phase experience, not by the pretest experience.

The results of Study 2 confirm and extend those of Study 1 . After viewing a KDE display that specified one or the other of two three-dimensional forms, 6-montholds again showed a preference significantly different from a corresponding baseline measure for the object not involved in the KDE display. Furthermore, this change in preference was observed even though the rhythm information that may have served as a basis for responding in Study 1 was not available. In Study 2, only the threedimensional form of the object involved in the KDE display could have linked the transformations of linear perspective provided by the display to the alternative sources of depth information available in the test displays.

\section{GENERAL DISCUSSION}

The results of the research reported here indicate that the perceptual phenomenon known as the kinetic depth effect emerges during the first half-year of life. In both Study 1 and Study 2, exposure to a KDE display of linear perspective transformations corresponding to a given three-dimensional form affected the relative preferences of 6-month-olds for that form as compared with another. The infants' preferences were affected in different ways in the two studies: in Study 1, relative attention to the form specified by the KDE display was reduced from the baseline measure (a "novelty response"), whereas in Study 2, it was enhanced from baseline (a "familiarity response"). The same sort of divergence was recently observed by E. J. Gibson and Walker (1984) in research on crossmodal perception during infancy. They speculated that the different directions of preference had to do with the differing extents of similarity between the habituation and test experiences in their two studies-the novelty response was evidenced when the stimulus presentations during habituation and test were very similar, and the familiarity response was evidenced when they were somewhat different. A number of researchers interested in modality interactions have also reported that infants exhibited novelty responses only when the "contexts" of habituation and testing were similar (Gottfried, Rose, \& Bridger, 1978; MacKay-Soroka, Trehub, Bull, \& Corter, 1982; Rolfe \& Day, 1981; Ruff, 1981). Thus, an explanation based on the degree of similarity between the habituation and test displays may account for the difference in the direction of the responses observed in the research 
reported here. In Study 1, the stimuli were presented undergoing the same sort of motion during habituation and test, namely rotation, whereas in Study 2, the stimuli were presented undergoing rotation during habituation but moving back and forth during test.

The directional difference discussed above may complicate the interpretation of the results of Studies 1 and 2 , but it does not alter the basic conclusion that 6-monthold infants can make use of the information related to three-dimensional form that is available in KDE displays. In each study, following exposure to a KDE display, infants' relative preference for the object involved in the display over another object was significantly different from a corresponding baseline measure. The preference shift in Study 1 might have been mediated by the perception of three-dimensional form from the KDE display or by sensitivity to a proximal cue of rhythm available in both the KDE and test displays. However, the preference shift in Study 2 could have been mediated only by the perception of three-dimensional form from the linear perspective transformations in the KDE display. The results thus indicate that, by 6 months, infants are sensitive to the kinetic depth cue of transformations of linear perspective and can appreciate depth relations as specified by this cue in isolation from other cues. Infants younger than 6 months of age may also be able to perceive threedimensional form from linear perspective transformations, but 4-month-olds did not consistently evidence this ability in the research described. The performance of some of the younger infants may have been compromised by an inability to perceive the three-dimensional structure of the test objects from binocular disparity information, since 4 months is a transitional age for disparity sensitivity. Additionally, 4 months could be a transitional age for sensitivity to transformations of linear perspective, too.

The finding that 6-month-olds are able to perceive threedimensional form from the isolated kinetic depth cue of linear perspective transformations contrasts with the difficulty they evidently have in perceiving threedimensional form when static and binocular information for object structure is available but kinetic information is not (see Ruff, 1978, 1982). Furthermore, Yonas et al.'s (1986) recent work suggests that the perception of threedimensional form from linear perspective transformations emerges either prior to or coincident with the perception of three-dimensional form from binocular disparity but in any case not after it. Thus, for infants, kinetic information may be the earliest or most heavily relied upon source of information regarding three-dimensional form. However, in practice, it would be extremely difficult to determine whether one or another of the various cues for three-dimensionality is actually primary, or in any sense "teaches" the others. The transfer paradigm employed here necessarily demands sensitivity to two different sources of depth information, and most other designs leave open the possibility that subjects may perform by discriminating or matching proximal stimuli rather than by genuinely perceiving three-dimensional structure. What is striking with regard to ontogeny, though, it that as best as it can be determined, true appreciation of threedimensional relations based on any and all combinations of the various cues for depth seems to become evident between 4 and 7 months (see Banks \& Salapatek, 1983; Granrud, 1986; Yonas \& Granrud, 1984). This age range is exactly the period during which infants are mastering visually guided reaching and object manipulation and beginning to experiment with self-produced locomotion. Whether these spatially related motor activities provide experience that gives rise to true depth perception or whether depth perception is preprogrammed to emerge coincident with them to facilitate their safe and effective deployment is an important developmental issue and a challenge for future research.

\section{REFERENCES}

Ball, W., \& Vurpillot, E. (1976). Perception of movement in depth in infancy. L'Année Psychologique, 76, 383-399.

Banks, M. S., \& Salapatek, P. (1983). Infant visual perception. In P. H. Mussen (Series Ed.), M. M. Haith \& J. J. Campos (Volume Eds.), Handbook of child psychology: Vol. 2. Infancy and psychobiology. New York: Wiley.

BirCH, E. E., GWIAZDA, J., \& HELD, R. (1982). Stereoacuity development for crossed and uncrossed disparities in human infants. Vision Research, 22, 507-513.

Birch, E. E., Shimojo, S., \& Held, R. (1985). Preferential-looking assessment of fusion and stereopsis in infants aged 1-6 months. Investigative Ophthalmology \& Visual Science, 26, 366-370.

BushNell, E. W., \& Roder, B. J. (1985). Recognition of color-form compounds by 4-month-old infants. Infant Behavior \& Development, 8, 255-268.

CAROLL, J. J., \& GiBson, E. (1981, April). Infants' differentiation of an aperture and an obstacle. Paper presented at the meeting of the Society for Research in Child Development, Boston.

CoHen, L. B., \& Younger, B. A. (1984). Infant perception of angular relations. Infant Behavior \& Development, 7, 37-47.

Fox, R., Aslin, R. N., Shea, S. L., \& Dumais, S. T. (1980). Stereopsis in human infants. Science, 207, 323-324.

Gibson, E. J., OWsley, C. J., Walker, A., MegaW-NyCe, J. (1979). Development of the perception of invariants: Substance and shape. Perception, 8, 609-619.

Gibson, E. J., \& WALKer, A. S. (1984). Development of knowledge of visual-tactual affordances of substance. Child Development, 55, 453-460.

GiBson, J. J. (1966). The senses considered as perceptual systems. Boston: Houghton Mifflin.

GiBson, J. J. (1979). The ecological approach to visual perception. Boston: Houghton Mifflin.

Gordon, F. R., \& YONAS, A. (1976). Sensitivity to binocular depth information in infants. Joumal of Experimental Child Psychology, 22, 413-422.

GotTfried, A. W., Rose, S. A., \& Bridger, W. H. (1978). Effects of visual, haptic, and manipulatory experiences on infants' visual recognition memory of objects. Developmental Psychology, 14, 305-312.

Granrud, C. E. (1986). Binocular vision and spatial perception in 4and 5-month-old infants. Journal of Experimental Psychology: $\mathrm{Hu}$ man Perception \& Performance, 12, 36-49.

Granrud, C. E., Yonas, A., Smith, 1. M., Arterberry, M. E., Glicksman, M. L., \& Sorknes, A. L. (1984). Infants' sensitivity to accretion and deletion of texture as information for depth at an edge. Child Development, 55, 1630-1636.

Johansson, G. (1974). Projective transformations as determining visual space perception. In R. B. MacLeod \& H. L. Pick (Eds.), Perception: Essays in honor of J. J. Gibson. Ithaca, NY: Cornell University Press. 
Kaufmann-Hayoz, R., Kaufmann, F., Stucki, M. (1986). Kinetic contours in infants' visual perception. Child Development, 57, 292-299.

Kellman, P. J. (1984). Perception of three-dimensional form by human infants. Perception \& Psychophysics, 36, 353-358.

KellmaN, P. J., \& SHORT, K. (1985, April). Infant form perception from perspective transformations. Poster presented at the meetings of the Society for Research in Child Development, Toronto.

Mackay-Soroka, S., Trehub, S. E., Bull, D. H., \& Corter, C. M. (1982). Effects of encoding and retrieval conditions on infants' recognition memory. Child Development, 53, 815-818.

OWSLEY, C. J. (1980). Perceiving object shape in early infancy: The role of kinetic information. Unpublished doctoral dissertation, Cornell University.

OWSLEY, C. J. (1983). The role of motion in infants' perception of solid shape. Perception, 12, 707-717.

Rolfe, S. A., \& DAY, R. H. (1981). Effects of the similarity and dissimilarity between familiarization and test objects on recognition memory in infants following unimodal and bimodal familiarization. Child Development, 52, 1308-1312.

RuFF, H. A. (1978). Infant recognition of the invariant form of objects. Child Development, 49, 293-306.

RUFF, H. A. (1980). The development of perception and recognition of objects. Child Development, 51, 981-992.

RUFF, H. A. (1981). Effect of context on infants' responses to novel objects. Developmental Psychology, 17, 87-89.

RUFF, H. A. (1982). Effect of object movement on infants' detection of object structure. Developmental Psychology, 18, 462-472.

WallaCh, H., \& O'ConNell, D. N. (1953). The kinetic depth effect. Journal of Experimental Psychology, 45, 205-217.

Yonas, A., ArTerberry, M. E., \& Granrud, C. E. (1986, April). Four-month-old infants' sensitivity to kinetic and binocular information for three-dimensional object shape. Poster presented at the International Conference on Infant Studies, Los Angeles.

Yonas, A., \& Granrud, C. E. (1984). The development of sensitivity to kinetic, binocular, and pictorial depth information in human infants. In D. Ingle, D. Lee, \& M. Jeannerod (Eds.), Brain mechanisms and spatial vision. Amsterdam: Martinus Nijhoff.

YonAs, A., \& PICK, H. L., JR. (1975). An approach to the study of infant space perception. In L. B. Cohen \& P. Salapatek (Eds.), Infant perception: From sensation to cognition (Vol. 2). New York: Academic Press.

\section{NOTES}

1. Further evidence that 4-month-olds do not respond according to the identity of the habituation stimulus in the type of procedure used is provided by data from an additional study conducted prior to Study 1. In this preliminary study, 20 4-month-olds were tested in a manner identical to that described for Study 1, except that no pretest trials were presented. The prediction was that the preferences exhibited by these infants on the posttest trials would be related to the stimulus they had seen during habituation. However, the durations of fixation on the posttest trial with the novel stimulus were equivalent to those on the trial with the familiar stimulus $(M s=15.5$ and $14.8 \mathrm{sec}$, respectively, $S D \mathrm{~s}=$ 11.5 and 11.4). Similarly, the mean posttest preference ratio computed for these infants did not depart significantly from the value of $.50 \mathrm{ex}-$ pected by chance $(M=.48, S D=.21$ ). Thus, the 4-month-olds in this initial study gave no indication of an ability to perceive three-dimensional form from linear perspective transformations. The posttest behavior of these infants suggested that individual infants may have had strong spontaneous preferences for one or the other of the stimulus objects, though, and these preferences may have obscured an effect of the habituation stimulus. Hence, the issue was reinvestigated in Study 1, with pretest trials incorporated into the procedure so that such idiosyncratic preferences could be negated in the data analyses.

2. This reversal across studies for the 6-month-olds raises the concern that analogous reversals might have occurred within Study 1 for the 4-month-olds. That is, perhaps the 4-month-olds perceived form from the habituation display, but some of the infants favored the novel stimulus on the posttest trials whereas others favored the familiar stimulus, yielding a null result overall. To address this concern, the data from the 4-montholds were further examined to determine whether or not their preference shifts were distributed bimodally. Difference scores representing the preference shifts from pre- to posttest were calculated and a histogram of these data was plotted. This analysis revealed an essentially normal curve with a slight positive skew but no bimodality $(M=.046$, $S D=.313$, median $=.001$ ). Seventy-five percent of the data points fell within one standard deviation of the mean and $95 \%$ fell within two standard deviations.

(Manuscript received April 1, 1985; revision accepted for publication July 13,1986 ). 